\title{
Ajuste psicológico y vida religiosa en adultos mayores*
}

Psychological Adjustment and Religious Life in Elderly People

Recibido: noviembre 23 de 2012 | Revisado: enero 1 de 2014 | Aceptado: enero 31 de 2014

\author{
ARMANDO RIVERA-LEDESMA ** \\ Universidad de Londres, México \\ María Montero-López LenA *** \\ Universidad Nacional Autónoma de México, México
}

doi:10.11144/Javeriana.UPSY13-3.apvr

Para citar este artículo: Rivera-Ledesma, A., \& Montero-López, M. (2014). Ajuste psicológico y vida religiosa en adultos mayores. Universitas Psychologica, 13(3), 895-906. http://dx.doi.org/10.11144/ Javeriana.UPSY13-3.apvr

* Agradecimientos: Agradecemos al Instituto Mexicano del Seguro Social y a la Congregación Cristiana por su apoyo y muy especialmente al Consejo Nacional de Ciencia y Tecnología por la beca No. 95177 otorgada al primer autor de este trabajo para la realización de estudios doctorales. Con amor, a la Sra. Consuelo Ledesma Galindo, 'Chelina':

** Coordinación de Investigación y Producción Científica. Correo electrónico: armandoriveral@ hotmail.com

**** Correo electrónico: monterol@unam.mx

\section{RES UMEN}

La presente investigación explora la relación entre la vida religiosa de las personas y el ajuste psicológico, con base en una muestra de 193 adultos mayores evangélicos de la comunidad de la Ciudad de México. El objetivo fue aportar evidencia empírica basada en una muestra latinoamericana, en relación con los controvertidos resultados en muestras anglosajonas, con respecto al papel de la vida religiosa como recurso adaptativo. Los resultados permiten concluir un papel marginal de la vida espiritual con respecto al desajuste psicológico, cuando es medido a través del establecimiento de una relación satisfactoria con Dios. Por otro lado, la presente investigación logró obtener fuertes asociaciones inversas, cuando la vida espiritual es asumida como satisfacción existencial o vital. Parece prevalecer una contribución marginal de la espiritualidad divina, en contraste con el importante papel que juega la espiritualidad vital o existencial en el desajuste psicológico de sujetos de la comunidad.

Palabras clave

Religión; espiritualidad; religiosidad; depresión; ansiedad

\section{A B S T R A C T}

This research explores the relationship between the religious life of the people and psychological adjustment based on a sample of 193 evangelical elderly living in the community of Mexico City. The aim was to provide empirical evidence based on Latin American sample, in relation to the disputed results in Anglo samples regarding the role of religious life as adaptive resource. The results show a marginal role in the spiritual life with respect to psychological maladjustment when measured through the establishment of a satisfactory relationship with God. Furthermore, this research was able to obtain strong inverse associations when the spiritual life is assumed as vital existential satisfaction. A marginal contribution of divine spirituality seems to prevail, in contrast to the important role that vital and existential spirituality plays in the psychological maladjustment in community subjects. Keywords

Religion; spirituality; religiosity; depression; anxiety 


\section{Antecedentes}

Blazer y Meador (Vahia et al., 2011), definen la religión como 'un sistema organizado de creencias, prácticas, rituales y símbolos, diseñados para facilitar la cercanía con lo sacro o lo trascendente', en tanto la espiritualidad es una entidad que abarca la religión, pero que se expande hasta un entendimiento de las respuestas a las preguntas últimas acerca de la vida, su sentido y su relación con lo sacro y lo trascendente. La religiosidad es esencialmente una experiencia de naturaleza social; se vive y experimenta en un nivel de experiencia social. En tanto la religión es objeto de la sociología, del análisis cultural, sociopolítico, antropológico, la religiosidad es un constructo que se juega en las condicionantes de la relación entre el yo y el otro; en la inmediatez social del individuo reglamentada por normas y códigos de conducta transmitidos a través del grupo. La religiosidad provee el marco conceptual de interpretación de la experiencia de lo divino; provee los medios para facilitar la ocurrencia de la experiencia espiritual e interpretarla cuando ya ha ocurrido. Operacionalmente, la religiosidad posee dos componentes: uno de orden cognitivo (conocimientos doctrinales o marco conceptual de interpretación) y otro de orden conductual (ritos y actividades religiosas tales como la asistencia a cultos, la participación en fiestas religiosas como el Sabbath o conductas como el rezar, orar, meditar, etc.).

Por otro lado, se ha destacado (Cook, 2004) la falta de acuerdo existente con respecto al constructo espiritualidad. Se ha sugerido que la naturaleza de la espiritualidad es singular, específica y personal (Emblen, 1992) y que incluye (Swinton, 2001 citado por Cook, 2004) aspectos de experiencia y creencia que son únicos del individuo. Así, este último propone la espiritualidad como una dimensión universal, potencialmente creativa y distintiva de la experiencia humana, que emerge, por un lado, dentro de la conciencia subjetiva interna de los individuos y, por otro, dentro de las comunidades, grupos sociales y tradiciones. Lo espiritual no es algo que se circunscriba a lo específicamente religioso. Así, la religiosidad es sustituida en las personas ateas por las reglas del amor, la responsabilidad, la camaradería, la solidaridad, la convivencia, la integración con el otro. La definición de Cook pone de relieve aspectos fundamentales, entre los que destaca la visión de lo espiritual como un problema de relación: 1. relación consigo mismo, 2. relación con el otro y 3. relación con lo divino. En el presente trabajo se asume que religión, religiosidad y espiritualidad son conceptos relacionados y distintos, y que la espiritualidad puede expresarse en relación con lo divino (lo sobrenatural, un ser superior como quiera que lo asuma el sujeto) y en relación con la vida misma (en la relación con el otro y la existencia).

Durante el siglo XX y el inicio del XXI se ha investigado ampliamente el papel de la religión, la vida religiosa y la vida espiritual, en relación con el ajuste y el bienestar psicológico, llevando a científicos de diversas latitudes a tratar de aportar evidencia empírica sobre la cualidad de dicha relación. Así, Bergin $(1983,1991)$ arribó a bajas correlaciones (0.09) entre religiosidad y salud mental. Gartner, Larson y Allen (1991), en una revisión del tema hallaron resultados muy ambiguos, siguiendo a Payne, Bergin, Bielema y Jenkins (1991). Larson et al. (1992) hallaron relaciones positivas en la mayoría de los estudios que revisaron, pero también relaciones negativas o neutrales en poco más de un cuarto de los estudios. Los resultados en general no han sido concluyentes, en parte, por las distintas definiciones aportadas en torno a conceptos centrales como espiritualidad, religiosidad y religión. De tales definiciones han derivado distintos instrumentos de medición, aplicados a distintas poblaciones; se ha sugerido que la orientación religiosa de los investigadores ha sido también una fuente de influencia en tales resultados (Pérez, Sandino \& Gómez, 2005).

Se ha señalado que cuando las personas atraviesan por intensas situaciones críticas en su vida, suelen recurrir también con mayor frecuencia a Dios y a ciertas conductas religiosas como la oración (Coleman et al., 2011; Kelley \& Chan, 2012) y, al parecer, estos recursos han resultado ser eficaces; el afrontamiento religioso ha sido reconocido como un importante recurso adaptativo (Pargament, 1997 citado por McConnell, Pargament, Ellison \& Flannelly, 2006). 
Distintos investigadores han reportado que la vida espiritual suele generar efectos positivos en enfermos crónicos, en casos de depresión (Cotton Larking, Hoopes, Cromer \& Rosenthal, 2005; Sorajjakool, Aja, Chilson, Ramirez-Jhonson \& Earll, 2008), VIH (Ironson, Stuetzle \& Fletcher, 2006), cáncer (McCoubrie \& Davis, 2006), entre otros padecimientos. En esta misma tesitura, Baldacchino y Buhagiar (2003) han destacado en concordancia con otros investigadores (Halm, Myers \& Bennetts, 2000 citados por Baldacchino \& Buhagiar, 2003; Koenig, George \& Siegler, 1988; Reed, 1987), que a pesar de que las personas pueden no ser altamente religiosas en su vida diaria, cuando llegan a enfermar suelen desarrollar prácticas religiosas como una forma de afrontamiento. Los investigadores consideran junto a otros (Bearon \& Koenig, 1990; Dreyer, 1996 citados por Baldacchino \& Buhagiar, 2003; Goddard, 1995), que esto es una consecuencia de la pérdida de control experimentada sobre la enfermedad y sus consecuencias; las personas necesitan encontrar un sentido y un propósito en esa experiencia y afrontar el estrés resultante; por ello, buscan ir más allá de sí mismos hasta alcanzar un poder superior o a Dios.

En un trabajo de metasíntesis, Hodge y Horvath (2011) analizaron 11 investigaciones cualitativas que indagaron en centros de salud acerca de la percepción de los pacientes con respecto a sus necesidades espirituales, encontrando lo siguiente: 1 . Expresaron la necesidad de comprender por qué ocurría la enfermedad o la muerte; necesitaban entender e interpretar los eventos que ocurrían en su vida. 2. En relación con Dios reportaron la necesidad de preguntar, culpar, defenderse, luchar, conciliarse con Dios, conocer el plan de Dios y aceptar o confiar en la voluntad de Dios. 3. Llevar a cabo prácticas espirituales (e. g., orar, leer la Biblia o libros espiritualmente orientados, alabar, escuchar música o mensajes alusivos a Dios, etc.), con el objetivo de fortalecer su relación con Dios. 4. Cumplir con sus obligaciones religiosas (e. g. comida kosher para los judíos o halaad para los musulmanes; evitar las transfusiones de sangre para los Testigos de Jehová). 5. Interactuar con personas de su misma fe (e. g. sacerdotes de su religión, amigos de su iglesia o de su misma religión; buscar el perdón de aquellos a quienes se ofendió o solicitar oración por sí mismo, etc.). 6. Con respecto a la interacción con el equipo de salud, los pacientes esperan un trato y una comunicación amable, con dignidad, respeto, empatía y cuidado, información amplia sobre su padecimiento y oportunidad de discutir el tratamiento.

Parece claro el importante papel que juega la vida religiosa y espiritual en las personas, cuando afrontan situaciones límite. Por ejemplo: Yi et al. (2006), en una muestra de 450 pacientes estadounidenses con VIH, encontraron una relación inversa entre síntomas depresivos y bienestar espiritual (medido a través de aspectos como la fe, consuelo y fortaleza en las propias creencias, sentido de significado, propósito y paz en la vida). Por otro lado, el afrontamiento religioso negativo y una actividad religiosa menos organizada estuvieron directa y significativamente asociados con síntomas depresivos, lo cual parece ser consistente con los hallazgos de Ano y Vasconcelles (2005). En otra investigación, Ironson, Stuetzle y Fletcher (2006), en una muestra de pacientes diagnosticados con VIH, encontraron que $45 \%$ de ellos presentó un incremento significativo (27\% “más” y 18\% “mucho más”), en religiosidad /espiritualidad, a partir del momento en que recibieron la noticia de ser positivos al VIH; 42\% no cambió y solo un $13 \%$ llegó a ser menos religiosos/espirituales (8\% “menos", 5\% “mucho menos"). Adicionalmente, los investigadores hallaron en su estudio longitudinal de cuatro años, que aquellos que mostraron los mayores incrementos en espiritualidad presentaron las menores pérdidas de células CD4 durante los cuatro años y un incremento más bajo en VL, en comparación con aquellos que mostraron un decremento espiritual. Sin embargo, en muestras conformadas por personas de la comunidad (sujetos no sometidos a problemas clínicos severos), diversos investigadores han reportado una relación inversa significativa entre variables religiosas y variables de desajuste psicológico como la depresión (Doolittle \& Farrel, 2004), ansiedad y hostilidad (e. g., Koenig \& Larson, 2001; Pargament, 1997, 2002; Schnittker, 2001 citados por Watlington \& Murphy, 2006). Watlington y Murphy (2006) hallaron correlaciones significativas 
de $-0.28,-0.29$ y 0 entre depresión y espiritualidad, participación religiosa y afrontamiento religioso, respectivamente. En una muestra colombiana, Pérez et al. (2005) no encontraron una asociación significativa entre depresión y prácticas religiosas; las únicas relaciones inversas se hallaron entre depresión y pertenencia a un grupo religioso, pero en una medida muy baja $(r=-0.16, p<0.01)$.

En una revisión, Koenig (2009) ha destacado que la religiosidad/espiritualidad ha mostrado una baja asociación con la depresión, pero que su valor resiliente entre aquellos sujetos religiosos es importante para salir de la depresión con mayor rapidez; también observó que los sujetos religiosos presentaban menos suicidios y más actitudes negativas en relación con el suicidio; varios estudios reportaban un efecto positivo que se reflejaba en menores grados de ansiedad. Se ha señalado (Coleman et al., 2011) que entre los recursos con los que cuenta la adultez mayor para afrontar el estrés y las pérdidas de esta etapa de la vida se encuentran las creencias y prácticas religiosas y espirituales, pero también se ha insistido en los hallazgos de diversos estudios con correlaciones menores aunque significativas entre estas y sus efectos.

Hackney y Sanders (2003), en un metaanálisis que comprendió 35 estudios, hallaron una correlación $r=0.1$, entre la salud mental y la espiritualidad. En otro trabajo metaanalítico, Smith, McCullough y Poll (2003 citados por Koenig, 2009) hallaron que en 147 estudios con 98975 sujetos existía una correlación negativa $r=-0.096$ entre espiritualidad y síntomas depresivos; esta asociación podría verse incrementada alrededor de $-\mathbf{0 . 1 5}$ en estudios donde las personas atravesaban situaciones críticas en su vida. Sin embargo, el seguimiento de 865 pacientes deprimidos permitió detectar que aquellos con una mayor asistencia semanal a la Iglesia, que oraban cotidianamente, leían las escrituras u otro material impreso religioso y que puntuaron alto en religiosidad intrínseca, se recuperaron de la depresión un 50\% más rápido que aquellos que no lo hacían.

De acuerdo con Pargament (1997 citado por McConnell et al., 2006), la prevalencia del afrontamiento religioso se encuentra entre el $20 \%$ y el $90 \%$, dependiendo de la muestra; es especialmente im- portante entre aquellas personas que afrontan eventos conflictivos en su vida (McConnell et al., 2006); se ha sugerido que a pesar de que las personas no sean esencialmente religiosas en la vida cotidiana, pueden recurrir a un afrontamiento religioso cuando atraviesan por situaciones de crisis tales como el curso de enfermedades (Quiceno \& Vinaccia, 2009; Koenig et al., 1988). En concordancia con esto, en un trabajo metaanalítico con 49 estudios, Ano y Vasconcelles (2005) evaluaron la relación entre afrontamiento religioso positivo y ajuste psicológico positivo al estrés, encontrando un efecto del orden de TE $=0.33($ IC 95\% = 0.3 - 0.35), indicativo de una relación positiva moderada entre el afrontamiento religioso positivo (e. g., apreciación religiosa benevolente, afrontamiento religioso colaborativo, búsqueda de soporte espiritual) y los resultados positivos a eventos estresantes (desarrollo espiritual, afectos positivos, mejor autoestima). Encontraron evidencia de que el afrontamiento religioso positivo está moderada e inversamente relacionado con un ajuste psicológico negativo (e. g., depresión, ansiedad, malestar psicológico [distress]; TE $=-0.12$ $(\mathrm{IC} 95 \%=-0.14-0.1)$. El afrontamiento religioso negativo (e.g. sentirse castigado por Dios, atribuir la situación a la obra del diablo, etc.) está modesta y positivamente asociado ( $\mathrm{TE}=0.22$, IC $95 \%=$ 0.19 - 0.24) con ajuste psicológico negativo al estrés (e. g., depresión, ansiedad, distrés). No se halló relación entre el afrontamiento religioso negativo y el ajuste positivo.

En México, Rivera-Ledesma y Montero-López (2005) investigaron la relación entre creencias y prácticas religiosas y espirituales (Holland, Kash, Passik, Gronert et al., 1998 citados por RiveraLedesma \& Montero-López, 2005) y el desajuste psicológico evidenciado por la depresión y la soledad en 125 sujetos de la comunidad con edades superiores a los 50 años (Media $=63.1$ años, $D E=$ 3.2) y mayoritariamente católicos $(N=93,74 \%)$. Los resultados mostraron que si bien la vida religiosa era importante para los adultos mayores, esta no se asoció significativamente con la depresión y la soledad; lo mismo se observó con el afrontamiento religioso y el soporte social religioso. En otra investigación relacionada, Montero-López y Rivera- 
Ledesma (2009), en una muestra de 122 sujetos con una media de edad de 60.9 años $(D E=8.24)$, en su mayoría católicos ( $N=106,87 \%)$, investigaron la relación entre la espiritualidad asociada a lo divino (Paloutzian \& Ellison, 1982 citados por MonteroLópez \& Rivera-Ledesma, 2009), el locus de control interno y el soporte social y el desajuste psicológico mostrado por la depresión, desesperanza, ideación suicida, soledad, ansiedad ante el envejecimiento, ansiedad ante la muerte, hallando que la única variable de desajuste que se asoció, y en una medida poco más que baja con la espiritualidad, fue la ansiedad ante el envejecimiento $(r=-0.26, p<0.01)$.

La aparente contradicción en los resultados con respecto al papel de la vida espiritual en el ajuste psicológico, parece deberse a distintos aspectos: 1) por un lado, a las muestras utilizadas: los sujetos que no atraviesan situaciones críticas parecen estar menos influidos por su vida espiritual religiosa, por el contario, aquellos que atraviesan situaciones límite presentan correlaciones más fuertes entre espiritualidad religiosa y ajuste psicológico. 2) Otro aspecto tiene que ver con la conceptualización de la espiritualidad/religiosidad y las variables que se asuman para medir el desajuste psicológico y la salud mental. Hackney y Sanders (2003) han profundizado en este problema. 3) El hecho de que la vida religiosa o espiritual de las personas sea multifacética, multifactorial (Gartner et al., 1991; Larson et al., 1992 citados por Hackney \& Sanders, 2003; Payne et al., 1991) hace difícil aprehender correctamente el impacto de estas importantes variables, mediante instrumentos de medición apropiados. 4) Otro aspecto, vinculado con el anterior, es el tipo de espiritualidad evaluada. Generalmente, se evalúa la espiritualidad que tiene que ver con lo divino, con lo eminentemente religioso (espiritualidad divina), dejando de lado aquella que tiene que ver con la existencia en si (espiritualidad existencial o vital).

A fin de aportar evidencia con respecto a esta problemática, en el presente trabajo se investiga la relación entre la espiritualidad divina (Satisfactoria relación con Dios), la espiritualidad existencial (Satisfactoria relación con la vida o satisfacción existencial), una Insatisfactoria relación con Dios y la vida (Insatisfacción existencial y con lo divino), el soporte social percibido y el desajuste psicológico representado por la depresión, ansiedad, desesperanza, soledad, ansiedad ante el envejecimiento, miedo a la muerte, enfermedad percibida e ideación suicida, en una muestra mexicana de sujetos adultos mayores cristianos evangélicos, de la comunidad (población no clínica). Se pretende evaluar si existen asociaciones significativas e importantes (mayores a $r=0.4$ ) entre las variables descritas.

\section{Método}

\section{Participantes}

La muestra fue no probabilística y estuvo conformada por 193 adultos mayores residentes de Ciudad de México, mayoritariamente cristianos evangélicos ( $N=188,97.9 \%$ ), elegidos por disponibilidad; la mayoría mujeres $(N=134,69.4 \%)$, con una edad media entre 60 años $(D E=7.5)$ y 60.8 años $(D E$ $=7.9$ ), para mujeres y hombres, respectivamente; 98 (51.3\%) no manifestaron tener compromiso marital alguno (ser casados o vivir en unión libre) y el resto, sí; 104 (54.4\%) no trabajaban; el 36.3\%, $27.5 \%, 11.4 \%$ y el $24.9 \%$ había terminado la primaria, secundaria, preparatoria y la universidad o estudios de posgrado, respectivamente. Todos los sujetos seleccionados cubrieron los siguientes criterios de inclusión: 1) Asistir de manera continua a una iglesia Cristiana-Evangélica. 2) Edad igual o mayor a 50 años. 3) Alfabetos. 4) Que aceptasen participar de manera informada y voluntariamente. 5) Capaces de contestar el cuestionario: conforme a las opciones de respuesta establecidas y al menos el 95\% de todas las preguntas.

\section{Instrumentos}

Se emplearon las siguientes escalas:

- Escala de Bienestar Espiritual ([EBE]; Paloutzian \& Ellison, 1982)

Citada y adaptada en adultos mayores mexicanos por Rivera-Ledesma y Montero-López (2007), mide bienestar espiritual a través de dos subescalas: una de Relación con Dios y otra de Satisfacción existencial. En esta investigación un análisis fac- 
torial reveló tres factores que explicaron el 51\% de la varianza ( $\alpha=0.79)$ : 1) Insatisfactoria relación con Dios y la vida: sentimientos de insatisfacción en la relación personal del sujeto con lo divino y la vida en general $(\alpha=0.65)$.2) Satisfactoria relación con Dios: sentimientos satisfactorios en la relación personal del sujeto con lo divino $(\alpha=0.66) .3)$ Satisfactoria relación con la Vida: sentimientos satisfactorios en la relación del sujeto con la vida misma en general $(\alpha=0.74)$.

- Escala de Soporte Social Percibido ([ESS]; Tagaya, Okuno, Tamura y Davis, 2000). Citada, traducida y adaptada en adultos mayores mexicanos en Rivera-Ledesma y Montero-López Lena (2007), con ( $\alpha=$ 0.91).

- World Health Organization Quality of Life Assessment, Brief, WHOQoL-Brief (WHOQoL-Group, 1998). Citada y adaptada en adultos mayores mexicanos en GonzálezCelis y Sánchez-Sosa (2002). Solo se utilizó la subescala de Enfermedad percibida ( $\alpha=$ 0.84).

- Escala de Depresión del Centro de Estudios Epidemiológicos ([CES-D]; Radloff, 1977). Con un $\alpha=0.89$.

- Hunter Opinions and Personal Expectations Scale ([HOPES]; Nunn, Lewin, Walton \& Carr, 1996). Citada, traducida y adaptada para adultos mayores mexicanos en RiveraLedesma y Montero-López (2007). Con un $\alpha=0.74$.

- Escala de Ansiedad ante el Envejecimiento ([AAE]; Lasher \& Faulkender, 1993). Citada, traducida y adaptada para adultos mayores mexicanos por Rivera-Ledesma y Montero-López (2007). Con un $\alpha=0.77$.

- Escala de Soledad en el Adulto Mayor ([IMSOL-AM]; Montero-López Lena \& RiveraLedesma, 2009). Con un $\alpha=0.89$.

- Inventario de Ansiedad de Beck ([IAB]; Comeche, Díaz \& Vallejo, 1995). Con un $\alpha=0.89$.

- Escala de Ansiedad ante la Muerte (Templer, 1969, 1970). Citada y adaptada en adultos mayores mexicanos en Rivera-Ledesma y Montero-López (2010). Con un $\alpha=0.83$.

- Escala de Ideación Suicida (Roberts, 1980). Citada en González-Forteza y Andrade Palos (1995). Con un $\alpha=0.79$.

\section{Procedimiento}

La presente investigación formó parte de un proyecto más amplio que consideró el empleo de un cuestionario constituido por 302 preguntas con opciones de respuesta en formato Likert. La información generada fue capturada directamente en el programa PASW 18 (SPSS); la información sociodemográfica y el contraste de hipótesis estadísticas (pruebas F, $t$ ) correlaciones $r$ de Pearson y análisis factorial, fueron desarrollados en el sistema PASW 18. El análisis del tamaño de los efectos para el caso de dos muestras independientes y sus intervalos de confianza al 95\% y ANOVA se realizó mediante la metodología sugerida por Morales (2012) para diferencias tipificadas; para el caso de las correlaciones se siguieron las sugerencias de Sánchez-Bruno y Borges (2005).

\section{Resultados}

\section{Asociaciones}

Las correlaciones obtenidas han sido descritas en la Tabla 1. En general parece ser, que el grado de satisfacción en relación con la vida y la existencia, reportado por la muestra, genera asociaciones inversas más frecuentes y más importantes con el desajuste psicológico, que el grado manifestado por la misma para la satisfacción generada por la relación que establece con lo divino. Para esta última variable, las correlaciones son en general bajas o no significativas, como ocurre con las variables de ansiedad general y miedo a la muerte y a la agonía, el miedo a los cambios físicos ocasionados por el envejecimiento, la ideación suicida, la enfermedad percibida y la carencia de propósito en la vida. Para el caso de la satisfacción existencial, variables como la ansiedad ante el envejecimiento, la depresión, la desesperanza, la soledad, la carencia de propósito en la vida y la enfermedad percibida, comparten el poseer asocia- 
ciones importantes e inversas con aquella, en tanto la actitud positiva ante el envejecimiento, el sentimiento de satisfacción con el self y la vida durante el proceso del propio envejecimiento, la esperanza y el soporte social percibido presentan asociaciones positivas, en su mayoría superiores a 0.5 .

Por otro lado, la insatisfacción existencial y con Dios se asoció positiva e importantemente (correlaciones entre 0.37 y 0.46) con el miedo a la muerte, la ansiedad ante el envejecimiento, insatisfacción con el self y la vida, la depresión, la desesperanza, la soledad, la enfermedad percibida, y negativamente con el soporte social percibido $(r=-0.4)$.

\section{Contraste de medias}

Los contrastes de medias que arrojaron resultados estadísticamente significativos aparecen en la Tabla
2. Se exploró la existencia de diferencias significativas en el grado de satisfacción en la relación con lo divino, según la frecuencia de asistencia a los eventos de la Iglesia a que acudía la muestra. Se hallaron diferencias importantes entre los que no asisten y los que al menos asisten una vez a la semana $(d=$ 0.94) y los que acuden 2 o 3 veces a la semana ( $d$ $=1.16$ ); asistir a la iglesia reporta mejores grados de satisfacción en la relación con Dios. El mismo efecto parece reportar el orar varias veces al día $(d$ $=0.97$ a 1.61). Sin embargo, el participar en algún ministerio de la iglesia parece aportar un efecto conservador $(d=0.29)$. La satisfacción existencial puede verse también beneficiada por el "platicar con Dios" $(d=0.97)$, cuando se lleva a cabo varias veces al día contra pocas veces a la semana; la diferencia entre orar varias veces al día contra una sola vez al día genera un efecto conservador $(d=$

TABLA 1

Correlaciones entre variables de ajuste psicológico y variables de espiritualidad existencial y divina

\begin{tabular}{|c|c|c|c|c|c|c|c|c|c|}
\hline \multirow[b]{3}{*}{ Variables de Ajuste Psicológico } & \multicolumn{3}{|c|}{$\begin{array}{l}\text { Espiritualidad vital o } \\
\text { Satisfacción existencial }\end{array}$} & \multicolumn{3}{|c|}{$\begin{array}{c}\text { Insatisfacción existencial } \\
\text { y con Dios }\end{array}$} & \multicolumn{3}{|c|}{$\begin{array}{l}\text { Espiritualidad divina o } \\
\text { Satisfacción con Dios }\end{array}$} \\
\hline & \multirow{2}{*}{$\begin{array}{l}\text { Zr de } \\
\text { Fisher }\end{array}$} & \multicolumn{2}{|c|}{ I.C. $95 \%$} & \multirow{2}{*}{$\begin{array}{l}\text { Zr de } \\
\text { Fisher }\end{array}$} & \multicolumn{2}{|c|}{ I.C. $95 \%$} & \multirow{2}{*}{$\begin{array}{l}\text { Zr de } \\
\text { Fisher }\end{array}$} & \multicolumn{2}{|c|}{ I.C. $95 \%$} \\
\hline & & Min & Max & & Min & Max & & Min & Max \\
\hline Ansiedad general & -0.38 & -0.52 & -0.23 & 0.32 & 0.18 & 0.46 & & & \\
\hline Miedo a la muerte & -0.32 & -0.46 & -0.18 & 0.37 & 0.22 & 0.51 & & & \\
\hline Miedo a la agonía & -0.22 & -0.37 & -0.08 & 0.34 & 0.2 & 0.49 & & & \\
\hline Ansiedad ante el envejecimiento & -0.58 & -0.72 & -0.43 & 0.4 & 0.26 & 0.54 & -0.32 & -0.46 & -0.18 \\
\hline AE- Actitud positiva & -0.37 & -0.51 & -0.22 & & & & -0.23 & -0.38 & -0.09 \\
\hline AE- Miedo a cambios físicos & -0.24 & -0.39 & -0.1 & 0.29 & 0.15 & 0.43 & & & \\
\hline AE- Insatisfacción con Self y la vida & -0.3 & -0.44 & -0.16 & 0.45 & 0.31 & 0.59 & -0.19 & -0.33 & -0.05 \\
\hline AE- Satisfacción con Self y la vida & -0.54 & -0.68 & -0.39 & 0.31 & 0.17 & 0.45 & -0.33 & -0.47 & -0.19 \\
\hline Depresión & -0.41 & -0.55 & -0.27 & 0.45 & 0.3 & 0.59 & -0.19 & -0.33 & -0.05 \\
\hline Desesperanza & -0.45 & -0.59 & -0.31 & 0.46 & 0.32 & 0.6 & -0.17 & -0.31 & -0.03 \\
\hline Ideación suicida & -0.31 & -0.45 & -0.17 & 0.28 & 0.13 & 0.42 & & & \\
\hline Esperanza & 0.58 & 0.43 & 0.72 & -0.29 & -0.43 & -0.15 & 0.29 & 0.15 & 0.43 \\
\hline Soledad & -0.56 & -0.7 & -0.42 & 0.42 & 0.28 & 0.57 & -0.17 & -0.31 & -0.03 \\
\hline S- Carencia de propósito en la vida & -0.66 & -0.8 & -0.52 & 0.42 & 0.28 & 0.57 & & & \\
\hline S- Inaceptación familiar & -0.38 & -0.52 & -0.23 & 0.34 & 0.2 & 0.48 & -0.18 & -0.32 & -0.04 \\
\hline Soporte social percibido & 0.36 & 0.22 & 0.51 & -0.4 & -0.54 & -0.26 & 0.24 & 0.1 & 0.39 \\
\hline Soporte social religioso & 0.32 & 0.18 & 0.46 & -0.18 & -0.32 & -0.04 & 0.31 & 0.17 & 0.45 \\
\hline Enfermedad percibido & -0.4 & -0.54 & -0.26 & 0.39 & 0.25 & 0.53 & & & \\
\hline
\end{tabular}

Solo se incluyen correlaciones estadísticamente significativas. Las correlaciones $\mathrm{r}$ de Pearson han sido transformadas mediante la Z de Fisher para la estimación de los intervalos de confianza (Sánchez-Bruno \& Borges, 2005); su interpretación es análoga a las correlaciones de Pearson. Se sombrean las correlaciones iguales o superiores a 0.35 .

Fuente: elaboración propia 
TABLA 2

Hábitos religiosos y satisfacción espiritualidad

\begin{tabular}{|c|c|c|c|c|c|c|c|c|c|c|c|}
\hline \multicolumn{12}{|c|}{$\begin{array}{l}\text { 1. Frecuencia de asistencia a los eventos de la iglesia. } \\
\text { Satisfactoria relación con Dios. } \\
\text { ANOVA: } F=3.751, g l=4, p=0.006 \text {. }\end{array}$} \\
\hline Frecuencia & $\mathrm{N}$ & M & $\sigma$ & $N$ & M & $\sigma$ & $\mathrm{T} / \mathrm{GH}$ & & $d$ & I.C. & $95 \%$ \\
\hline No asiste / 1 vez por semana & 12 & 12.92 & 2.53 & 90 & 14.68 & 1.75 & $p=0.012$ & & 0.94 & 0.33 & 1.54 \\
\hline No asiste / 2,3 veces semana & 12 & 12.92 & 2.53 & 85 & 14.98 & 1.63 & $p=0.002$ & & 1.16 & 0.55 & 1.76 \\
\hline \multicolumn{12}{|c|}{$\begin{array}{l}\text { 2. Frecuencia con que el sujeto platica con Dios } \\
\text { Satisfactoria relación con Dios. } \\
\text { ANOVA: } F=22.92, g l=2, p=0\end{array}$} \\
\hline $\begin{array}{l}\text { Varias veces al día vs. Pocas veces a la } \\
\text { semana }\end{array}$ & 135 & 15.2 & 1.33 & 11 & 13 & 1.61 & $p=0.003$ & & 1.61 & 1 & 2.23 \\
\hline Varias veces al día vs. Una vez al día & 135 & 15.2 & 1.33 & 47 & 13.6 & 2.3 & $p=0$ & & 0.97 & 0.64 & 1.3 \\
\hline \multicolumn{12}{|l|}{$\begin{array}{l}\text { Insatisfacción existencial y con Dios. } \\
\text { ANOVA: } F=5.55, g l=2, p=0.005 \text {. }\end{array}$} \\
\hline Varias veces al día vs. Una vez al día & 135 & 8.37 & 2.5 & 47 & 9.79 & 2.86 & $p=0.004$ & & 0.54 & 0.21 & 0.88 \\
\hline \multicolumn{12}{|l|}{$\begin{array}{l}\text { Satisfacción existencial. } \\
\text { ANOVA: } F=22.92, g l=2, p=0\end{array}$} \\
\hline $\begin{array}{l}\text { Varias veces al día vs. Pocas veces a la } \\
\text { semana }\end{array}$ & 135 & 10.19 & 1.8 & 11 & 8.45 & 1.5 & $p=0.012$ & & 0.97 & 0.36 & 1.59 \\
\hline Varias veces al día vs. Una vez al día & 135 & 10.19 & 1.8 & 47 & 9.06 & 2.25 & $p=0.002$ & & 0.58 & 0.25 & 0.92 \\
\hline \multicolumn{12}{|c|}{ 3. Participación o no en algún ministerio de la iglesia } \\
\hline & \multicolumn{3}{|c|}{ Participa } & \multicolumn{3}{|c|}{ No Participa } & & & & & \\
\hline Área & $N$ & M & $\sigma$ & $N$ & $M$ & $\sigma$ & $t$ & $g l$ & $d$ & I.C. & $95 \%$ \\
\hline Insatisfacción existencial y con Dios & 76 & 7.8 & 1.93 & 117 & 9.4 & 2.85 & $4.63 * *$ & 190.55 & 0.63 & 0.34 & 0.92 \\
\hline Satisfactoria relación con Dios & 76 & 15 & 1.57 & 117 & 14.48 & 1.93 & $2.05^{*}$ & 181.31 & 0.29 & 0 & 0.58 \\
\hline Satisfacción existencial & 76 & 10.3 & 2.04 & 117 & 9.5 & 1.89 & $2.77 * *$ & 191 & 0.41 & 0.12 & 0.7 \\
\hline
\end{tabular}

$* p \leq 0.05 ; * * \leq 0.01 \mathrm{~T} / \mathrm{GH}=$ Fórmula de Tukey o Games Howell, según exista o no homocedasticidad Fuente: elaboración propia

0.58), algo similar ocurre con el participar o no en algún ministerio de la iglesia; participar arroja un efecto moderado $(d=0.41)$. Por su parte, el efecto de orar varias veces al día contra el hacerlo una sola vez, reporta un efecto conservador $(d=0.54)$.

\section{Discusión}

Los resultados descritos aportan evidencia acerca de la relación entre espiritualidad divina y espiritualidad existencial o vital y el desajuste psicológico, en una muestra no clínica de sujetos de la comunidad, todos pertenecientes a una congregación cristiana evangélica de Ciudad de México. Los hallazgos parecen ser consistentes con la literatura generada en otras latitudes (Bergín, 1983, 1991; Doolittle \& Farrel, 2004; Hackney \& Sanders, 2003; Pérez et al., 2005; Vahia et al., 2011; Watlington \& Murphy, 2006). La relación entre la espiritualidad divina y el desajuste psicológico no reporta correlaciones iguales o mayores a 0.4 ; existen bajas correlaciones negativas con respecto a variables como depresión $(-0.19)$, desesperanza y soledad (ambas de -0.17) o no significativas como en el caso de la ansiedad general y el miedo a la muerte y la agonía. Estos resultados parecen destacar un papel marginal de la vida espiritual con respecto al desajuste psicológico, cuando es medido a través del establecimiento de una Relación satisfactoria con Dios (espiritualidad definida como el establecimiento de una relación personal con Dios; "los sentimientos, actos y experiencias de los hombres individuales en su solitud cuando se perciben a sí mismos en relación con lo que consideran lo divino" (James, 1988, p. 34), 
lo cual aporta nueva evidencia a la reportada por Rivera-Ledesma y Montero-López $(2005,2008)$ en muestras católicas. Al parecer, católicos y cristianos presentan el mismo tipo de relación con respecto al desajuste psicológico; ni unos ni otros logran beneficiarse de una satisfactoria relación con lo divino en variables como depresión, ansiedad, soledad, desesperanza y ansiedad ante la muerte.

Dado que la evidencia reportada por los investigadores de lo espiritual aporta bastos antecedentes positivos acerca de la espiritualidad divina o satisfacción con Dios, como recurso adaptativo en situaciones de intenso estrés (e. g., enfermedades crónicas), parecería claro hipotetizar que la espiritualidad divina juega un papel más instrumental, en términos adaptativos, que trasformador, en términos de un desarrollo consistente de la personalidad en torno a los valores formativos de la doctrina religiosa del creyente. Eso podría explicar la diferencia en los resultados reportados en la literatura. Con todo, la espiritualidad divina parece jugar un papel importante al asociarse inversamente con la ansiedad ante el envejecimiento, tanto en lo general, como en el grado de satisfacción con el self y la vida $(r=-0.32$ y -0.33 , respectivamente), lo cual también es consistente con los hallazgos de Rivera-Ledesma y Montero-López (2009) en sujetos católicos; ello apoyaría la hipótesis de que la vida espiritual puede ser importante como recurso adaptativo ante las situaciones de estrés usualmente asociadas a la vejez (Blazer, 2000; Koenig, Cohen, Blazer \& Meador 1992; Stevens, 2000).

Estos datos parecen encontrar respaldo en el hecho de que el viejo, como lo señaló Erikson (1983), necesita integrar positivamente su vida; esta "integración del Yo es la aceptación del propio y único ciclo de vida" (p. 241); la insatisfacción producto del fracaso de esta integración conduciría al adulto mayor a la desesperación, al desajuste psicológico (cf., Fortner \& Neimeyer, 1999; Rappaport et al., 1993). Una solución contraria, la integración, generaría un estado de bienestar emocional producto de la integración yoica de los hechos de la vida en un todo conciliado lleno de un sentido positivo. Esta parece ser la tarea fundamental de la vejez, y es posible que en esta constelación de elementos, la relación con lo divino ocupe un papel secundario o accesorio; podría ser que lo divino jugase un papel adaptativo al servicio de una necesidad superior que sería la conciliación e integración de la propia vida.

Por otro lado, la presente investigación logró obtener fuertes asociaciones inversas (superiores a $r=0.4$ ), entre la vida espiritual cuando es asumida como Satisfacción existencial o vital y el desajuste psicológico; las asociaciones entre esta espiritualidad vital y la soledad, la desesperanza, la ansiedad ante el envejecimiento y dentro de esta última la satisfacción con el self y la vida, superan un coeficiente de $r=0.5$. Como Koenig (2009) ha sugerido, la espiritualidad está muy influida por variables asociadas al soporte social; la espiritualidad vital o satisfacción existencial parece estar claramente determinada por la relación que el sujeto establece con el otro y consigo mismo, como puede verse en la Escala de Bienestar Espiritual (Paloutzian \& Ellison, 1982), empleada aquí. En la literatura se cuenta con abundante evidencia acerca de la relación entre el soporte social percibido y el bienestar psicológico.

En otro sentido, una Insatisfactoria relación con Dios y la Vida (factor que mezcla ambas dimensiones) se asoció positivamente al desajuste psicológico y específicamente con la ansiedad ante el envejecimiento y dentro de esta con la insatisfacción con el self y la vida, la depresión, la desesperanza, la soledad, la carencia de propósito en la vida y el soporte social percibido, todas con correlaciones iguales o superiores a 0.4. Estos datos parecen cerrar el círculo con respecto a la importancia de lo espiritual en el ajuste psicológico: una satisfactoria vida espiritual en relación con la vida y la existencia parece asociarse a menores niveles de desajuste psicológico; una vida insatisfactoria con la existencia y lo divino, por el contrario, parecería contribuir al desajuste psicológico.

Ahora bien, de los hábitos religiosos investigados en relación con la espiritualidad, ha sido posible aportar evidencia acerca del efecto positivo de asistir a los eventos de la iglesia en el desarrollo de una satisfactoria relación con Dios. Por otro lado, el orar o platicar con Dios también contribuye a este efecto positivo cuando se lleva a cabo varias veces al día, y aunque en menor medida, también 
puede beneficiar una satisfactoria relación con la vida y un menor grado de insatisfacción existencial y con Dios. Esta última variable puede a su vez ser positivamente influida al participar activamente en un ministerio de la iglesia. Estos datos permiten apreciar en alguna medida el valor de la religiosidad como aportadora de los recursos disponibles para el desarrollo espiritual.

La presente investigación aportó evidencia con respecto a la contribución marginal de la espiritualidad divina, por un lado, y el importante papel que juega la espiritualidad vital o existencial, por otro, al desajuste psicológico. Es importante considerar que estas conclusiones han sido extraídas de una muestra no clínica, con sujetos de la comunidad. Sería importante replicar esta investigación en poblaciones clínicas con sujetos inmersos en enfermedades de difícil pronóstico, con el objetivo de evaluar la importancia de la espiritualidad bajo tales exigencias adaptativas.

\section{Referencias}

Ano, G. G., \& Vasconcelles, B. E. (2005). Religious coping and psychological adjusment to stress: A meta-analysis. Journal of Clinical Psychology, 61(4), 461-480.

Baldacchino, D. R., \& Buhagiar, A. (2003). Psychometric evaluation of the Spiritual Coping Strategies Scale in English, Maltese, back-translation and bilingual versions. Journal of Advanced Nursing, 42(6), 558-570.

Bergin, A. E. (1983). Religiosity and mental health: A critical reevaluation and meta-analysis. Professional Psychology: Research and Practice, 14(2), 170-184.

Bergin, A. E. (1991). Values and religious issues in psychotherapy and mental health. American Psychologist, 46(4), 394-403.

Blazer, D. G., II (2000). Spirituality, aging and depression. En J. A. Thorson, James (Ed.), Perspectives on spiritual well-being and aging (pp. 161-169). Springfield, IL: Charles C. Thomas, Ltd.

Coleman, P. G., Carare, R. O., Petrov, I., Forbes, E., Saigal, A., Spreadbury, J. H., Yap, A., et al. (2011). Spiritual belief, social support, physical functioning and depression among older people in Bulgaria and Romania. Aging $\mathcal{E}$ Mental Health, 15(3), 327 333.

Cook, C. C. H. (2004). Addiction and spirituality. Addiction, 99(5), 548-549.

Comeche, M., Díaz, M. \& Vallejo, M. (1995). Cuestionarios, inventarios y escalas: ansiedad, depresión y habilidades sociales. Madrid: Fundación Universidad-Empresa.

Cotton, S., Larking, E., Hoopes, A., Cromer, B. A., \& Rosenthal, S. L. (2005). The impact of adolescent spirituality on depressive symptoms and health risk. Adolescent Health, 36(6), 529.e7-529.e14.

Doolittle, B. R., \& Farrel, M. (2004). The association between spirituality and depression in an urban clinic. Journal of Clinical Psychiatry, 6(3), 114-118.

Emblen, J. D. (1992). Religion and spirituality defined according to current use in nursing literature. Journal of Professional Nursing, 8(1), 41-47.

Erikson, E. H. (1983). Infancia y sociedad. Buenos Aires: Ediciones Hormé.

Fortner, B. V., \& Neimeyer, R. A. (1999). Death anxiety in older adults: A quantitative review. Death studies, 23(5), 387-412.

Gartner, J., Larson, D. B., \& Allen, G. D. (1991). Religious commitment and mental health: A review of the empirical literature. Journal of Psychology and Theology, 19, 6-25.

González-Forteza, C., \& Andrade, P. (1995). La relación de los hijos con sus progenitores y sus recursos de apoyo: correlación con la sintomatología depresiva y la ideación suicida en los adolescentes mexicanos. Salud Mental, 18(4), 41-48.

González-Celis, R. A. L., \& Sánchez-Sosa, J. J. (2002). Efectos de intervención de un programa de promoción a la salud sobre la calidad de vida de los ancianos. Tesis Doctoral, Universidad Nacional Autónoma de México, Facultad de Psicología, Ciudad Universitaria, México, D. F.

Hackney, C. H., \& Sanders, G. S. (2003). Religiosity and mental health: A meta-analysis of recent studies. Journal for the Scientific Study of Religion, 42(1), 43-55.

Hodge, D. R., \& Horvath, V. E. (2011), Spiritual needs in health care settings: A qualitative meta-synthesis of clients' perspectives. Social Work, 56(4), 306-316. 
Ironson, G., Stuetzle, R., \& Fletcher, M. A. (2006). An increase in religiousness/spirituality occurs after HIV diagnosis and predicts slower disease progression over 4 years in people with HIV. Journal of General Internal Medicine, 21, S62-S68.

James, W. (1988). Las variedades de la experiencia religiosa. Estudio de la naturaleza humana. Buenos Aires: Hyspamerica Ediciones Argentina.

Kelley, M. M., \& Chan, K. T. (2012). Assessing the role of attachment to God, meaning, and religious coping as mediators in the grief experience. Death Studies, 36(3), 199-227.

Koenig, H. G. (2009). Research on religion, spirituality, and mental health: A review. The Canadian Journal of Psychiatry, 54(5), 283-291.

Koening, H. G., Cohen, H. J., Blazer, D. G., \& Meador, K. G. (1992). Religious coping and depression in elderly hospitalized medically ill men. American Journal of Psychiatry, 149(12), 1693-1700.

Koenig, H. G., George, L. K., \& Siegler, I. C. (1988). The use of religion and other emotion-regulating coping strategies among older adults. The Gerontologist, 28(3), 303-310.

Larson, D. B., Sherrill, K. A., Lyons, F. C., Craigie, F. C., Thielman, M. A., Greenwold, M. A., \& Larson, S. S. (1992). Associations between dimensions of religious commitment and psychiatry and mental health reported in the American Journal of Archives of General Psychiatry: 1978-1989. American Journal of Psychiatry, 149(4), 557-559.

McConnell, K. M., Pargament, K. I., Ellison, C. G., \& Flannelly, K. J. (2006). Examining the links between spiritual struggles and symptoms of psychology in a national sample. Journal of Clinical Psychology, 62(12), 1469-1484.

McCoubrie, R., \& Davis, A. (2006). Is there a correlation between spirituality and anxiety and depression in patients with advanced cancer? Supportive Care in Cancer, 14(4), 379-385.

Montero-López, M., \& Rivera-Ledesma, A. (2009). Variables con alto valor adaptativo en el desajuste psicológico del adulto mayor. Journal of Behavior, Health Eु Social Issues, 1(1), 59-67.

Morales, V. P. (2012). El tamaño del efecto (effect size): análisis complementarios al contraste de medias. Universidad Pontificia Comillas, Madrid/Uni- versidad Rafael Landivar, Guatemala. Consultado el 5 de noviembre del 2012 en http://www. upcomillas.es/personal/peter/investigacion/ Tama\%F1oDelEfecto.pdf

Paloutzian, R., \& Ellison, C. (1982). Loneliness, spiritual well-being and the quality of life. En D. Peplau \& D. Perlman D. (Eds.), Loneliness: A sourcebook of current theory, research and therapy (pp. 224-235). New York: John Wiley \& Sons.

Pérez, A., Sandino, C., \& Gómez, V. O. (2005). Relación entre depresión y práctica religiosa: un estudio exploratorio. Suma Psicológica, 12(1), 77-86.

Payne, I. R., Bergin, K. A., Bielema, K. A., \& Jenkins, P. H. (1991). Review of religion and mental health prevention and the enhancement of psychosocial functioning. Prevention in Human Services, 9(2), 11-40.

Quiceno, J. M., \& Vinaccia, S. (2009). La salud en el marco de la psicología de la religión y la espiritualidad. Diversitas. Perspectivas en Psicología, 5(2), 321-336.

Radloff, L. S. (1977). The CES-D Scale: A self-report depression scale for research in the general population. Applied Psychological Measurement, 1(3), 385-401.

Rappaport, H., Fossler, R. J., Bross, L. S., \& Gilden, D. (1993). Future time, death anxiety, and life purpose among older adults. Death Studies, 17(4), 369-379.

Rivera-Ledesma, A., \& Montero-López, M. (2005). Espiritualidad y religiosidad en adultos mayores mexicanos. Salud Mental, 28(6), 51-58.

Rivera-Ledesma, A., \& Montero-López, M. (2007). Modelo de intervención racional emotivo para la promoción del ajuste psicológico del adulto mayor en un contexto religioso [Tesis Doctoral]. Universidad Nacional Autónoma de México, Facultad de Psicología, México.

Rivera-Ledesma, A., \& Montero-López, L. M. (2008). Estructura de pérdidas en la adultez mayor: una propuesta de medida. Salud Mental, 3(6), 461-468.

Rivera-Ledesma, A., \& Montero-López, L. M. (2009). Variables con alto valor adaptativo en el desajuste psicológico del adulto mayor. Journal of Health and Social Issues, 1(1), 59-67.

Rivera-Ledesma, A., \& Montero-López, L. M. (2010). Propiedades psicométricas de la Escala de Ansie- 
dad ante la Muerte de Templer en sujetos mexicanos. Diversitas. Perspectivas en Psicología, 6(1), 135-140.

Sánchez-Bruno, A., \& Borges, Á. (2005). Transformación $Z$ de Fisher para la determinación de intervalos de confianza del coeficiente de correlación de Pearson. Psicothema, 17(1), 148-153.

Sorajjakool, S., Aja, V., Chilson, B., Ramirez-Jhonson, J., \& Earll, A. (2008). Disconnection, depression, and spirituality: A study of the roll of spirituality and meaning aim the lives of individuals with severe depression. Pastoral Psychology, 56(5), 521-532.

Stevens, D. D. (2000). Spiritualy, self-transcendence and depression in young adults whit AIDS (immune deficiency). Dissertation Abstracts International, Section B: Sciences $\mathcal{E}$ Engineering, 61(2-B), 785.

Vahia, I. V., Depp, C. A., Palmer, B. W., Fellows, I., Golshan, S., Thompson, W., \& Allison, M., et al. (2011). Correlates of spirituality in older women. Aging and Mental Health, 15(1), 97-102.

Watlington, C. G., \& Murphy, C. M. (2006). The roles of religion and spirituality among African American survivors of domestic violence. Journal of Clinical Psychology, 62(7), 837-857.

Yi, M. S., Mrus, J. M., Wade, T. J., Ho, M. L., Hornung, R. W., Cotton, S., Peterman, A. H., et al. (2006). Religion, spirituality, and depressive symptoms in patients with HIV/AIDS. Journal of General Internal Medicine, 21(Supl. 5), S21-27. 